\title{
Formulação e caracterização físico-química de geleias convencional e dietética de mandacaru e umbu
}

\author{
Formulation and physicochemical characterization of conventional and diet jelly of mandacaru and \\ umbu
}

Formulación y caracterización fisicoquímica de mermeladas dietéticas y convencionales de mandacaru y umbu

Recebido: 08/06/2021 | Revisado: 16/06/2021 | Aceito: 19/06/2021 | Publicado: 04/07/2021

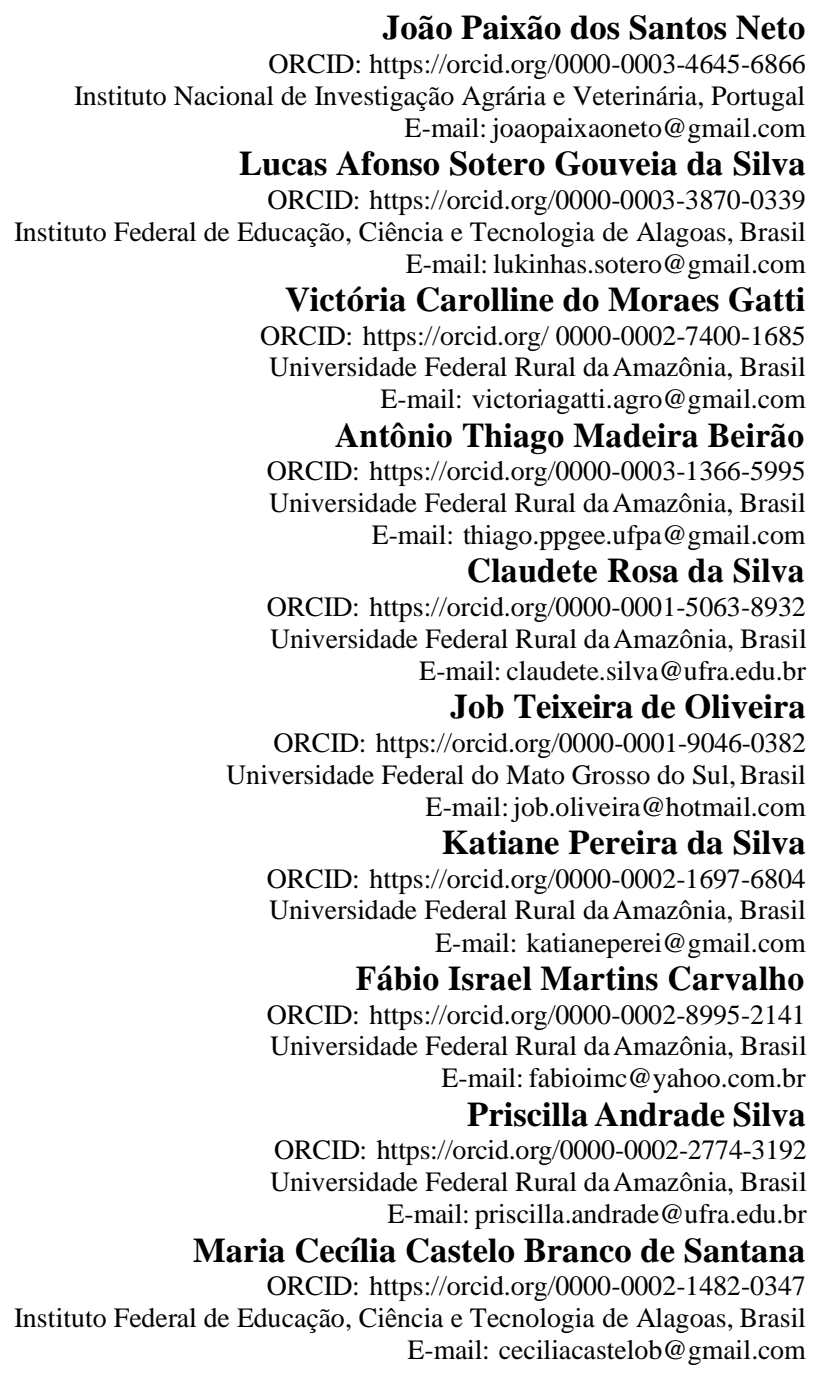

\section{Resumo}

A geleia é considerada um produto de destaque em escala industrial, devido à boa aceitação pelos consumidores. O objetivo deste estudo foi desenvolver geleia convencional e dietética mista de umbu e mandacaru. As geleias foram elaboradas através da formulação padrão e dietética, uma com sacarose e a outra com xilitol, polpa de umbu e de mandacaru, pectina cítrica e ácido cítrico. Após 30 dias de armazenamento em temperatura ambiente foram avaliados os parâmetros físico-químicos. Os resultados obtidos foram submetidos à análise de variância pelo software Agroestat. Os valores médios das características físico-químicas dos frutos de umbu e mandacaru obtiveram valores satisfatórios para produção de geleia. A geleia convencional se enquadra nos aspectos da legislação em vigor. A geleia dietética não atendeu o padrão de identidade e qualidade e apresentou a presença de sinérese.

Palavras-chave: Caatinga; Geleia; Qualidade físico-química. 


\begin{abstract}
Jelly is considered a prominent product on an industrial scale, due to its good acceptance by consumers. The aim of this study was to develop conventional and mixed dietary jelly from umbu and mandacaru. The jellies were made using the standard and dietary formulation, one with sucrose and the other with xylitol, pulp of umbu and mandacaru, citric pectin and citric acid. After 30 days of storage in the environment, the physicochemical parameters were evaluated. The results obtained were subjected to analysis of variance by the Agroestat software. The average values of the physicochemical characteristics of the fruits of umbu and mandacaru obtained satisfactory values for jelly production. Conventional jelly falls within the aspects of the legislation in force. The diet jelly did not meet the identity and quality standards and showed the presence of syneresis.
\end{abstract}

Keywords: Caatinga; Jelly; Physicochemical quality.

\title{
Resumen
}

La gelatina se considera un producto destacado a escala industrial, debido a su buena aceptación por parte de los consumidores. El objetivo de este estudio fue desarrollar gelatina dietética convencional y mixta a partir de umbu y mandacaru. Las jaleas se elaboraron utilizando la formulación estándar y dietética, una con sacarosa y la otra con xilitol, pulpa de umbu y mandacaru, pectina cítrica y ácido cítrico. Después de 30 días de almacenamiento en el medio ambiente, se evaluaron los parámetros físico-químicos. Los resultados obtenidos se sometieron a análisis de varianza mediante el software Agroestat. Los valores promedio de las características fisicoquímicas de los frutos de umbu y mandacaru obtuvieron valores satisfactorios para la producción de gelatina. La gelatina convencional se enmarca dentro de los aspectos de la legislación vigente. La gelatina dietética no cumplió con los estándares de identidad y calidad y mostró la presencia de sinéresis.

Palabras clave: Caatinga; Jaleas; Calidad fisicoquímica.

\section{Introdução}

O bioma da caatinga, exclusivamente brasileiro, ocupa uma área de aproximadamente $844.453 \mathrm{~km}^{2}$, equivalente a $11 \%$ do território nacional. As plantas desse bioma enfrentam longos períodos de seca, que provocam alterações no metabolismo bioquímico ocasionando modificações nos níveis carboidratos, proteínas e outras substâncias orgânicos principalmente a síntese de compostos bioativos (Drumond et al. 2016).

O mandacaru (Cereus jamacaru DC.) é considerado maior variedade de Cactaceae, encontrada na Região Nordeste, pertence ao gênero Cereus. Os frutos são basiformes e, em geral, grandes e vermelhos, de polpa adocicada, e muito apreciados por animais e pelo homem (Silva et al. 2020). Entretanto, é um fruto bastante perecível, frágil, com vida útil curta, representando um gargalo para sua comercialização in natura (Reis et al. 2020). A composição do fruto de mandacaru depende do estádio de maturação e das condições edafoclimáticas entre outros fatores. Segundo Nascimento et al. (2011) o mandacaru possui teores de proteínas $(1,80-2,35 \%)$, lipídeos $(1,08-1,98 \%)$, carboidratos $(9,76-9,86 \%)$, minerais $(0,43$ - 0,64\%), sólidos solúveis totais (10,30 - 12,03 ${ }^{\circ}$ Brix), $\mathrm{pH}(4,40$ - 4,93), acidez titulável total $(0,26$ - 0,32\% ácido cítrico) e água $(85,82-86,28 \%)$.

O umbuzeiro (Spondias tuberosa Arruda) é uma frutífera tropical com alta de produção de frutos sob às condições de estresse hídrico e é vastamente encontrado na caatinga (Drumond et al. 2016) sendo considerado uma fonte alternativa de renda aos pequenos agricultores do Semiárido nordestino (Gonçalves, 2018). O umbu apresenta características sui generis e diversos benefícios à saúde (Moura et al. 2013), pois em sua composição nutricional detêm a presença de vitaminas (B1, B2, A, C e niacina), minerais (cálcio, fósforo e ferro), alto teor de taninos $\left(126,27 \mathrm{mg} .100 \mathrm{~g}^{-1}\right.$ ) e antioxidantes (Vidigal et al. 2011). De acordo com Melo e Andrade (2010) os estádios de maturação de umbu semi-maduro (11,07 mg.100g-1) possui maior quantidade de ácido ascórbico do que o maduro $\left(9,38 \mathrm{mg} \cdot 100 \mathrm{~g}^{-1}\right)$, já o teor de carotenoides totais ocorre o inverso $\left(3,02 \mu \mathrm{g} \cdot \mathrm{g}^{-1}\right.$ nos frutos maduros e $1,70 \mu \mathrm{g} \cdot \mathrm{g}^{-1}$ nos semi-maduros).

Uma alternativa viável para os frutos de mandacaru e umbu é o processamento agroindustrial, uma possibilidade para conservação é a produção de geleia (Reis et al. 2020). No entanto, a preocupação global com a qualidade de vida foi oportuna para o desenvolvimento de produtos dietéticos desde que possuam características sensoriais similares aos produtos convencionais. Desse modo o emprego dos polióis, nomeadamente do xilitol, conferem textura aos alimentos, contribuem para o valor nutricional e sensoriais desejáveis (Viana et al. 2015). 
Devido às características sui generis dos frutos de mandacaru e umbu, este estudo tem como objetivo elaborar duas formulações de geleia, convencional e dietética, visando atender a exigência de diferentes perfis de diferentes consumidores, como também caracterizar a composição físico-química das geleias.

\section{Material e Métodos}

Os frutos do umbuzeiro e do mandacaru foram coletados no município de Piranhas, localizado na mesorregião do Sertão Alagoano, tem uma altitude aproximada de $88 \mathrm{~m}$ e coordenadas geográficas de $9^{\circ} 37^{\prime} 38^{\prime \prime}$ de latitude sul e $37^{\circ} 45^{\prime} 25^{\prime \prime}$ de longitude oeste.

Posteriormente, os frutos foram selecionados em estádio maduro provenientes da safra 2018/19 e lavados em água corrente e em seguida imersos em solução clorada (10 ppm de hipoclorito de sódio por 30 minutos), após foi feito o enxágue. Em seguida, os frutos de umbus foram cozidos inteiros com a casca durante aproximadamente 8 minutos após o início da fervura seguidamente foi removida a água numa peneira de aço inoxidável e retiradas as sementes. No preparado da polpa do mandacaru foi realizada a remoção do epicarpo dos frutos. Assim, foram obtidas as polpas integrais de umbu e mandacaru.

Para a elaboração da geleia dietética e convencional (F1), foi utilizado $48,5 \%$ de sacarose, 50\% de polpa (sendo, $25 \%$ de umbu e $25 \%$ de mandacaru) e $0,5 \%$ de pectina cítrica (alto teor de metoxilação) e $1 \%$ de ácido cítrico, Tabela 1 . A formulação da geleia dietética (F2) foi utilizado 22\% de xilitol (Figura 1), 38,5\% de polpa de mandacaru, 38,5\% de polpa de umbu, 0,5\% de pectina e $1 \%$ de ácido cítrico, Tabela 1 . A quantidade de $22 \%$ de xilitol, foi encontrada a partir dos pré-testes realizados, verificou que ocorreu a melhor formação de geleificação e não teve a dificuldade de dissolução deste poliol, conforme podemos verificar na Figura 1. Prontamente, procedeu o processo tecnológico de obtenção da geleia do tipo extra, de acordo com Viana et al. (2011). Após a obtenção das geleias estas foram acondicionadas em embalagens de vidro, previamente esterilizadas a $100^{\circ} \mathrm{C} / 15$ min com capacidade para $500 \mathrm{~g}$, fechadas com tampa de metal e invertidas por 10 minutos. O tratamento térmico foi efetuado em banho-maria a $100^{\circ} \mathrm{C}$ por 15 minutos, em seguida foram resfriadas por adição de água fria por 15 minutos e posteriormente armazenadas em local fresco na ausência de luminosidade.

Tabela 1 - Formulações de geleias de umbu e mandacaru tradicional e dietética

\begin{tabular}{ccc}
\hline Ingredientes & F1 $(\%)$ & F2 $(\%)$ \\
\hline Polpa de mandacaru & 25,00 & 38,25 \\
Polpa de umbu & 25,00 & 38,25 \\
Sacarose & 48,50 & - \\
Xilitol & - & 22,00 \\
Pectina & 0,50 & 0,50 \\
Ácido citríco & 1,00 & 1,00 \\
\hline
\end{tabular}

Fonte: Autores. 
Figura 1 - Pré-testes com as diferentes concentrações de xilitol. (A) Geleia com 49,5\% de xilitol; (B) Geleia com 33\% de xilitol e (C) Geleia com $22 \%$ de xilitol.

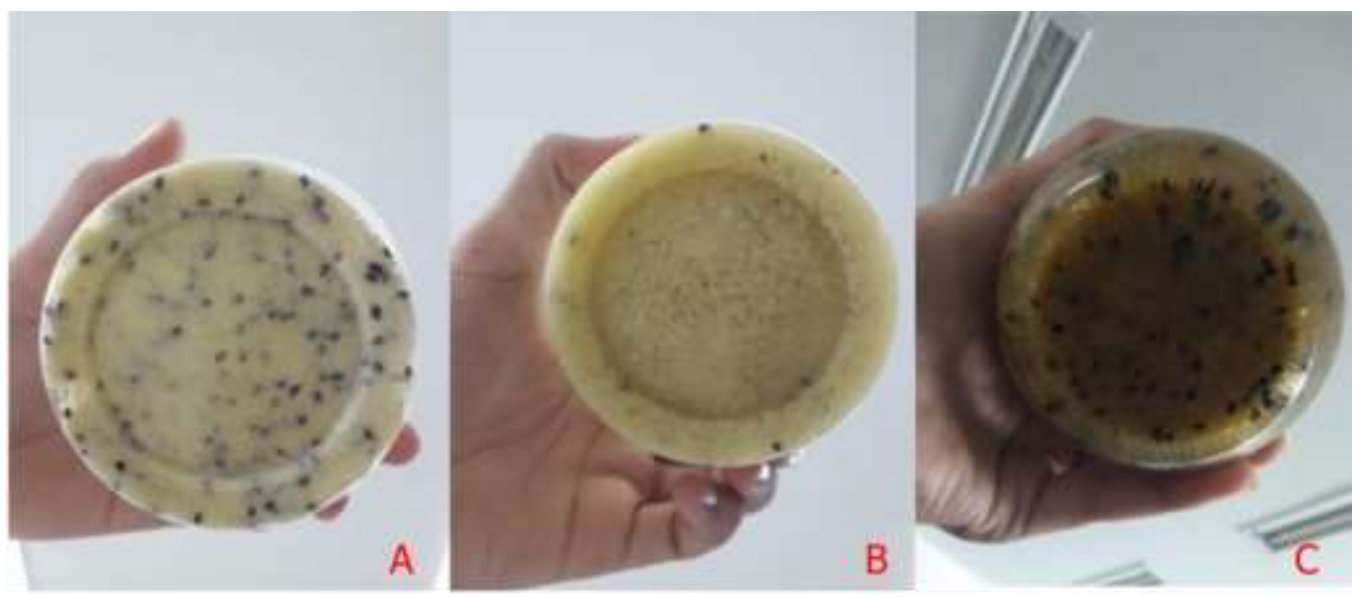

Fonte: Autores.

A qualidade inicial no momento da colheita foi avaliada em 10 amostras de cada fruto, foi determinado: o peso através de balança analítica, altura e largura mensurado pelo paquímetro, densidade pelo deslocamento da coluna de água em proveta. Na polpa dos frutos e nas geleias foram analisados: o pH, teor de sólidos solúveis totais (SST), acidez total titulável (ATT), todas em triplicatas; umidade e cinzas, em quadruplicata, de acordo com a metodologia descrita pelo Instituto Adolfo Lutz (IAL, 2008).

Os dados obtidos nas análises físicas e físico-químicas dos frutos de mandacaru e umbu foram submetidos a estatística das variáveis respostas com o auxílio do software Agroestat (Barbosa; Maldonado, 2010). Após análise de variância as médias foram comparadas pelo Teste de Tukey, com $\mathrm{p} \leq 0,05$.

\section{Resultados e Discussão}

As características físicas dos frutos de mandacaru e umbu estão apresentados na Tabela 2.

Tabela 2 - Características físicas dos frutos de mandacaru e umbu.

\begin{tabular}{ccccc}
\hline Fruto & $\begin{array}{c}\text { Peso } \\
(\mathrm{g})\end{array}$ & $\begin{array}{c}\text { Comprimento } \\
(\mathrm{cm})\end{array}$ & $\begin{array}{c}\text { Largura } \\
(\mathrm{cm})\end{array}$ & $\begin{array}{c}\text { Firmeza } \\
(\mathrm{N})\end{array}$ \\
\hline Mandacaru & $78,32 \pm 35,19$ & $9,70 \pm 11,13$ & $4,31 \pm 0,58$ & $70,11 \pm 23,84$ \\
Umbu & $19,58 \pm 4,96$ & $3,04 \pm 0,34$ & $2,77 \pm 0,77$ & $33,88 \pm 16,63$ \\
\hline
\end{tabular}

Resultados expressos em base úmida $(\mathrm{n}=3)$. Fonte: Autores.

O fruto do mandacaru apresentou formato elipsoide, já o umbu formato arredondado. Em relação as variáveis físicas dos frutos de mandacaru e umbu, observamos que os frutos de mandacaru apresentaram maiores valores medidos. De acordo com Melo et al. (2017) o mandacaru do agreste do estado da Paraíba apresentou valores para comprimento, largura e peso 10,69 cm, 5,93 cm e 191,24 g, respectivamente, maiores do que os encontrados no presente estudo. Esta variação pode ser devido a fatores climáticos, que podem ter aumentado o tamanho dos frutos.

Costa et al. (2015) avaliaram a biometria dos frutos de umbu da região do semiárido brasileiro, relataram valores para comprimento 2,53 a 4,35 cm e de largura variando de 2,46 a 4,30 cm. Estes valores corroboram com os valores encontrados na Tabela 2 .

Acerca do peso de mandacaru, segundo Melo et al. (2017) reportaram média de 191,24 g, enquanto Santos Neto et al. (2019) média de 108,12 g. Os pesos diferem dos encontrados no presente trabalho, que apresentam a média de 78,32g. O teor de 
água no solo influencia na produtividade das frutíferas e no tamanho e padronização dos frutos. As análises destes frutos foram feitas em locais diferentes e com regimes de precipitações diferentes, o que pode ter influenciado no tamanho e peso dos frutos.

Para umbu, segundo Souza et al. (2018) o peso foi equivalente a 10,74g, o que também difere dos valores médios de 19,58g encontrados na Tabela 2. O peso dos frutos é uma característica importante durante a comercialização in natura dos frutos, conforme afirma Santos et al. (2010), frutos mais pesados são maiores, tornando-se mais atrativos para os consumidores.

Würz et al., (2020) relatam que a firmeza da polpa é um dos parâmetros qualitativos mais importantes para o fruto, pois está relacionada com a capacidade de conservação dos frutos em pós-colheita. A perda da firmeza, aliada à mudança da cor da casca, é a transformação mais característica que ocorre durante o amadurecimento. Os frutos de mandacaru e umbu estavam maduros conforme valores dispostos na Tabela 2.

Logo, as variáveis físicas dos frutos de mandacaru e umbu apresentaram alta variabilidade dos valores (Tabela 2). De acordo com Melo et al. (2017) e Santos Neto (2019) que avaliaram os frutos de mandacaru colhidos em diferentes estados do nordeste, Paraíba e Alagoas, demonstraram que para os parâmetros físicos existe uma variação dos dados encontrados de acordo com a região. Justamente, foi observado também esta variação nos aspectos físicos dos frutos de mandacaru e umbu analisados neste estudo.

As características químicas da polpa de mandacaru e umbu estão dispostas na Tabela 3.

Tabela 3 - Características físico-químicas da polpa de mandacaru e umbu.

\begin{tabular}{lccccc}
\hline Polpa & $\begin{array}{c}\text { Sólidos } \\
\text { solúveis } \\
\text { totais } \\
(\mathrm{SST})(\%)\end{array}$ & $\mathrm{pH}$ & $\begin{array}{c}\text { Acidez } \\
\text { Total } \\
\text { Titulável } \\
(\text { ATT }) \\
(\%)\end{array}$ & $\begin{array}{l}\text { Umida- } \\
\text { de }(\%)\end{array}$ & $\begin{array}{c}\text { Cinzas } \\
(\%)\end{array}$ \\
\hline Mandacaru & $11,00 \pm 0,08$ & $4,67 \pm 0,05$ & $0,13 \pm 0,01$ & $86,11 \pm 1,97$ & $0,44 \pm 0,01$ \\
Umbu & $8,10 \pm 0,08$ & $2,67 \pm 0,05$ & $0,94 \pm 0,02$ & $88,35 \pm 0,18$ & $0,35 \pm 0,04$ \\
\hline
\end{tabular}

Resultados expressos em base úmida $(\mathrm{n}=3)$. Fonte: Autores.

O valor médio do teor de sólidos solúveis totais (SST) no mandacaru foi de 11,00\%. Este dado corrobora com o encontrado por Santos Neto et al. (2019) de 11,03\%. Entretanto, Bahia et al. (2010) identificaram teores de SST de mandacaru de 14,1\%. Contudo, Melo et al. (2017) reportaram que os teores de SST de mandacaru, aumentaram com o avanço da maturação evoluindo de 9,93 a 14,30\%, para os estádios de maturação verde claro e totalmente vermelho. Os valores encontrados para os frutos de umbu, concorda com Lima e Silva (2016) referem que a variabilidade genética dos frutos de umbu influência diretamente os parâmetros físico-químicos, e exibiram valores de SST de 7,5\%.

O potencial hidrogeniônico $(\mathrm{pH})$ dos frutos de mandacaru e umbu indicam que são frutos ácidos, todavia contribuem para redução da capacidade de desenvolvimento microbiano. De acordo com Santos Neto et al. (2019), o valor de pH encontrado no trabalho sobre características físico-químicas de frutos de mandacaru (Cereus Jamacaru P. Dc.) foi de 3,73 um resultado de pH menos ácido que os valores encontrados no presente estudo, que foram de 4,67.

$\mathrm{Na}$ literatura para os frutos de umbu segundo Silva et al. (2017), detectou em torno de 2,35. Dantas \& Silva (2017) identificaram o valor médio de $\mathrm{pH}$ na ordem de 3,43 segundo os resultados das análises físico-químicas do fermentado de umbu. Os valores corroboram com os valores encontrados de 2,67.

Os valores de acidez total titulável (ATT) da Tabela 3, consentem com Melo et al. (2017) que obtiveram 0,35\% de ATT e Santos Neto (2019) de 0,22\% de ATT, ambos para os frutos de mandacaru. No entanto, os frutos de umbu de acordo com 
Ferreira et al. (2015) observaram valores de AT entre 0,41 a 0,58\%. No estudo desenvolvido por Santos et al. (2017), ao analisarem três estádios de maturação de umbu, obtiveram os referidos valores de ATT, verde $(1,08 \%)$, intermediário $(0,85 \%)$ e maduro $(0,92 \%)$.

A umidade, disposta na Tabela 3, demonstra o alto percentual de água livre em ambos frutos. Bahia et al. (2010) avaliaram a umidade dos frutos de mandacaru e obtiveram um valor de 83,29\%. Oliveira et al. (2011) mencionam que a elevada quantidade de água livre não favorece a sua conservação, tendo a necessidade de armazenamento sob refrigeração.

Os resultados de cinzas (Tabela 3) expressam a quantidade de minerais totais. Os valores de cinzas encontrados por Santos Neto et al. (2017) foi de 0,42\% semelhante a este estudo (0,48\%) para mandacaru. Já, para umbu segundo Paula et al. (2012) foi em torno de $0,41 \%$, valor maior que o encontrado neste estudo, que apresentou $0,35 \%$.

A avaliação da qualidade físico-química das geleias, disponíveis na Tabela 4.

Tabela 4 - Características químicas de geleia convencional (F1) e dietética (F2) de mandacaru e umbu.

\begin{tabular}{cccccc}
\hline $\begin{array}{c}\text { Sólidos } \\
\text { solúveis } \\
\text { totais } \\
\text { (SST) }(\%)\end{array}$ & $\mathrm{pH}$ & $\begin{array}{c}\text { Acidez } \\
\text { Total } \\
\text { Titulável } \\
(\text { ATT })(\%)\end{array}$ & $\begin{array}{c}\text { Umi- } \\
\text { dade } \\
(\%)\end{array}$ & $\begin{array}{c}\text { Cinzas } \\
(\%)\end{array}$ \\
\hline F1 & $71,00 \pm 1,0 \mathrm{a}$ & $3,03 \pm 0,05 \mathrm{a}$ & $0,49 \pm 0,03 \mathrm{a}$ & $29,91 \pm 1,67 \mathrm{~b}$ & $0,31 \pm 0,04 \mathrm{~b}$ \\
F2 & $40,22 \pm 0,1 \mathrm{~b}$ & $3,07 \pm 0,05 \mathrm{a}$ & $0,51 \pm 0,03 \mathrm{a}$ & $62,30 \pm 5,08 \mathrm{a}$ & $0,42 \pm 0,04 \mathrm{a}$ \\
\hline
\end{tabular}

Médias seguidas por letras iguais, na coluna, não diferem entre si, ao nível de 5\% de significância. Fonte: Autores.

A partir dos resultados, observamos diferença estatística para os teores de SST, umidade e cinzas nas geleias. Conforme Gava (2008), as condições ótimas para a formação do gel estão próximas do pH 3,2 e foi possível a formação do gel, entretanto a manutenção não ocorreu como esperado. Foi identificado que a geleia dietética não enquadrou-se com a quantidade mínima de SS definida pela legislação (Brasil, 2001), pois o teor de SST deve ser maior ou igual a 65\%, desta forma diverge do padrão de identidade e qualidade de geleia. Além disso, a geleia dietética apresentou sinérese, ou seja, a exsudação do líquido da geleia.

As características químicas da geleia, corroboram em alguns parâmetros com o estudo de Souza et al. (2018) sobre a elaboração e avaliação da qualidade de geleia de umbu e mangaba, que apresentaram valores de $\mathrm{pH}$ de 2,63, valor próximo ao encontrado pelo presente trabalho de 3,03 e 3,07 para geleia convencional e dietética respectivamente. Os teores de SST estão entre $63,90 \%$ e $67,30 \%$ o que está próximo ao resultado encontrado para o teor de SS de geleia convencional $(71,00)$, já os resultados dos teores de SST de geleia dietética foram relativamente menores (40,22\%). Lemos et al. (2019) apresentou teores entre $0,77 \%$ e $0,80 \%$ para ATT.

Os teores de Umidade para geleia convencional e dietética encontrados foram de $29,91 \%$ e $62,30 \%$ respectivamente, corroboram em partes com os resultados encontrados por Souza et al. (2018) de $24,95 \%$ e $29,57 \%$. Os teores de cinza são de $0,31 \%$ e $0,42 \%$, valores relativamente distantes dos encontrados por Lemos et al. (2019) com teores de $0,66 \%$ e $0,76 \%$.

\section{Considerações Finais}

Os valores médios das características físico-químicas dos frutos de umbu e mandacaru obtiveram valores satisfatórios para produção de geleia.

A geleia convencional se enquadra nos aspectos da legislação em vigor.

A geleia dietética não atendeu o padrão de identidade e qualidade e apresentou a presença de sinérese. 


\section{Agradecimentos}

O desenvolvimento deste trabalho contou com apoio do Programa Institucional de Iniciação Científica e de Desenvolvimento Tecnológico e Inovação (PIBIC/PIBITI) do Instituto Federal de Alagoas pelas bolsas concedidas aos autores.

\section{Referências}

Bahia, E. V. A., Morais, L. R. V., Silva, M. P., Lima, O. B. V., Santos, S. F. (2010). Estudo das características físico-químicas do fruto do mandacaru (Cereus jamacaru P.DC.) cultivado no sertão Pernambucano. Anais do Congresso de Pesquisa e Inovação da Rede Norte Nordeste de Educação Tecnológica (CDROM). IFAL, 5.

Barbosa, J. C., Maldonado, J. R. W. (2010). AgroEstat: sistema para análises estatísticas de ensaios agronômicos. Versão 1.0. Jaboticabal: Departamento de Ciências Exatas.

Brasil. Resolução Normativa CTA $n^{o}$. 15/78. (2001). Compêndio da Legislação de Alimentos, ABIA.

Costa, F. R., Rêgo, E. R., Rêgo, M. M., Neder, D. G., Silva, S. M. \& Schunemann, A. P. P. (2015) - Análise biométrica de frutos de umbuzeiro do semiárido Brasileiro. Bioscience Journal 31(3) p. 682 - 690. https://doi.org/10.14393/BJ-v31n3a2015-22844.

Dantas, C. E. A., Silva, J. L. A. (2017). Fermentado alcoólico de umbu: produção, cinética de fermentação e caracterização físico-química. Holos, 2,108 - 121. 10.15628/holos.2017.4506.

Drumond, M. A., Aidar, S. T., Nascimento, C. E., Oliveira, V. R. (2016). Umbuzeiro: avanços e perspectivas. Petrolina: Embrapa Semiárido. ISBN 978-857035-570-6.

Ferreira, L. B., Duarte, A. B., Martins, J. C., Ferreira, A. F. \& Mizoutsi, G. P. (2015). Caracterização físico- -química de frutos de umbuzeiro (Spondias tuberosa Arr. Câm.) de Janaúba-MG. In: Anais do $1^{\circ}$ Congresso Brasileiro de Processamento Mínimo e Pós-Colheita de Frutas, Flores e Hortaliças, Aracaju, Brasil.

Gava, A. J. (2008). Princípios da Tecnologia de Alimentos. Nobel.

Gonçalves, P. C. (2018). O mandacaru não floresceu: a ciência positivista a serviço do combate à seca de 1877-1879. Hist. cienc. saúde - Manguinhos 25 http://dx.doi.org/10.1590/s0104-59702018000200012.

Lemos, D. M., Rocha, A. P. T., Gouveia, J. P. G., Oliveira, E. N. A., Sousa, E. P., Silva, S. F. (2019). Elaboração e caracterização de geleia prebiótica mista de jabuticaba e acerola. Braz. J. Food Technol., 22, e2018098. https://doi.org/10.1590/1981-6723.09818.

Lima, M. A. C., Silva, S. M. (2016). Qualidade e conservação pós-colheita. In: Drumond, M. A., Aidar, S. T., Nascimento, C. E. S., Oliveira, V. R. (Eds.). Umbuzeiro: avanços e perspectivas. Petrolina: Embrapa Semiárido, p. 176 - 215.

Maia, M. C. A., Galvão, A. P. G. L. K., Modesta, R. C. D., Pereira JR, N. (2008). Avaliação Sensorial de Sorvetes à Base de Xilitol. Ciência e Tecnologia de Alimentos, 28, p. 151 - 152. https://doi.org/10.1590/S0101-20612008000100021.

Melo, E. A., Andrade, R. A. M. S. (2010). Compostos bioativos e potencial antioxidante de frutos do umbuzeiro. Alimentos e Nutrição, 21 (3) p. 453 - 457

Melo, R. S., Silva, S. M., Sousa, A. S. B., Lima, R., Dantas, A. L., Dantas, R. L., Figueiredo, V. M. A. (2017). Maturação e qualidade de frutos de mandacaru (Cereus jamacaru P. DC.) de diferentes bioclimas do estado da Paraíba. Agropecuária Técnica (UFPB), 38 (3), 160.

Minim, V. P. R. (2013). Análise sensorial: estudos com consumidores. 3. ed. Viçosa: Editora UFV, 332 p.

Moura, F. T. et al. (2013). Evolução do Crescimento e da Maturação de Frutos de Cajazeira (Spondias mombin L). Proceedings of The Interamerican Society for Tropical Horticulture, 47, 231 - 233.

Nascimento, V. T., Moura, N. P., Vasconcelos, M. A. S., Maciel, M. I. S., Albuquerque, U. P. (2011). Chemical characterization of native wild plants of dry seasonal forests of the semi-arid region of northeastern Brazil. Food Research International, 44, p. 2112 - 2119. https://doi.org/10.1016/j.foodres.2010.12.024.

Paula, B., Carvalho F., Celso D., Matta, V. M., Menezes, J. S., Lima, P. C., Pinto, C. O., Conceição, L. E. M. G. (2012). Produção e caracterização físico-química de fermentado de umbu. Ciência Rural (UFSM. Impresso), 42, p. 1688 - 1693. https://doi.org/10.1590/S0103-84782012000900027.

Reis, C. G., Figueirêdo, R. M. F., Queiroz, A. J. M., Amadeu, L. T. S., Lima, T. L. B., Costa, P. S. (2020). Geleias de melão com casca eenriquecida comsementes de mandacaru. Revista Verde, 15:4 p.414-421. https://doi.org/10.18378/rvads.v15i4.7715.

Ribeiro, F. W., Rodrigues, C. C., Bert, M. P. S., Silva, A. C., Peixoto, N. (2020). Elaboração de geléias de umbu nas formulações padrão e zero açúcar: análise sensorial e índice de aceitabilidade. Sci. Elec. Arch. 13 (6). http://dx.doi.org/10.36560/1362020991.

Santos, D. C. A., Melo, G. K. S. S., Silva, W. A., Moura, F. J. A., Silvério, M. L., Correia, J. M., Bezarra, V. S. (2021). Produção, análise físico-química e sensorial de geleia de abiu (Pouteria caimito) com chia. Brazilian Journal of Development, 7, 7118 - 7133. 10.34117/bjdv7n1-482.

Santos, M. B., Cardoso, R. L., Fonseca, A. A. O. \& Conceição, M. N. (2010) - Caracterização e qualidade de frutos de umbu-cajá (Spondias tuberosa X S. mombin) provenientes do Recôncavo Sul da Bahia. Revista Brasileira de Fruticultura, 32(4), 1089 - 1097.

Santos Neto, J. P., Silva, V. D. N., Silva, P. A., Santos, Y. M. P., Monteiro, P. H. S., Silva, L. A. S. G. (2019). Características Físico-Químicas de Frutos de Mandacaru (Cereus Jamacaru P. Dc.) Cultivados no Sertão Alagoano. Revista Craibeiras de Agroecologia, 4 , e7741. https://www.seer.ufal.br/index.php/era/article/view/7741. 
Research, Society and Development, v. 10, n. 8, e4710816955, 2021

(CC BY 4.0) | ISSN 2525-3409 | DOI: http://dx.doi.org/10.33448/rsd-v10i8.16955

Silva, M. I., Silva, G. R., Alves, J. E. A., Martins, J. N. (2017). Caracterização físico-química da polpa de umbu in natura. Reunião Regional da SBPC no Cariri - 02 a 06 de maio de 2017 - URCA - Cariri/CE. Disponível em: http://www.sbpcnet.org.br/livro/cariri/resumos/1894.pdf.

Silva, N. R., Almeida, A. A., Mimura, A. M. S. (2020). O uso de Mandacaru (Cereus jamacaru) na alimentação de bovinos no interior do Piauí. ACSA, Patos$P B, 16,104$ - 110. http://dx.doi.org/10.30969/acsa.v16i2.1220.

Souza, H.R.S., Carvalho, M. G., Santos, A. M., Ferreira, I. M., Silva, A. M. O. (2018). Compostos bioativos e estabilidade de geleia mista de umbu (Spondias tuberosa arr. c.) e mangaba (Hancornia speciosa g.). Revista Brasileira de Higiene e Sanidade Animal 12, 236 - 248. http://dx.doi.org/10.5935/19812965.20180023

Souza, H. R. S., Santos, A. M., Ferreira, I. M., Silva, A. M. O., Nunes, T. P., Carvalho, M. G. (2018). Elaboração e avaliação da qualidade de geleia de umbu (Spondias Tuberosa Arr. C.) e mangaba (HancorniaSpeciosa G.) com alegação funcional. Segur.Aliment. Nutr., $25,104 \quad-113$. http://dx.doi.org/10.20396/san.v25i3.8652496.

Viana, E. S., Oliveira, M. E., Carvalho, L. D., Oliveira, L. A. (2011). Processamento de umbu-cajá, variedade suprema, na forma de geleia dietética e convencional. Circular Técnica n. 102. www.embrapa.gov.br.

Viana, E. S., Mamede, M. E. O., Reis, R. C., Carvalho, L. D. Fonseca, Mércia Damasceno. (2015). Desenvolvimento de geleia de umbu-cajá convencional e dietética. Revista Brasileira de Fruticultura, 37, 708 - 717. https://doi.org/10.1590/0100-2945-018/14.

Vidigal, M. C. T. R., Minim, V. P. R., Carvalho, N. B., Milagres, M. P., Gonçalves, A. C. A. (2011). Effect of a health claim on consumer acceptance of exotic brazilian fruit juices: Açaí (Euterpe oleracea Mart.), camu-camu (Myrciaria dubia), cajá (Spondias lutea L.) and umbu (Spondias tuberosa Arruda). Food Research International, 44 (7), 1988 - 1996. https://doi.org/10.1016/j.foodres.2010.11.028.

Würz, D. A., Kowal, A. N., Fagherazzi, A. F., Santos, G., Leite, L. (2020). Efeito da aplicação foliar de silício nos aspectos produtivos e de qualidade de frutos de morangueiro. Revista Eletrônica Científica da UERGS, 6 (2), 144 - 149. http://dx.doi.org/10.21674/2448-0479.62.144-149. 